\title{
Capillary electrophoresis-mass spectrometry analysis of trehalose-6-phosphate in Arabidopsis thaliana seedlings
}

\author{
T. L. Delatte • H. Schluepmann • S. C. M. Smeekens • \\ G. J. de Jong • G. W. Somsen
}

Received: 7 December 2010 /Revised: 4 February 2011 /Accepted: 22 February 2011 /Published online: 12 March 2011

(C) The Author(s) 2011. This article is published with open access at Springerlink.com

\begin{abstract}
Trehalose-6-phosphate (T6P) is an intermediate in the plant metabolic pathway that results in trehalose production. T6P has been shown to inhibit the sucrose nonfermenting-1-related protein kinase 1, which is a major regulator of metabolism. The quantitation of T6P has proven difficult due to the complexity of the plant matrix and the low abundance of T6P in plant tissues. The aim of this work was to develop a quantitation method for T6P present in Arabidopsis tissues, with capillary electrophoresis (CE) coupled to electrospray ionization-mass spectrometry (MS) with a sheath liquid (SL) interface. The CE-MS method was first optimized with respect to T6P signal intensity and separation of isomers by studying the composition of the background electrolyte (BGE) and SL. The use of triethylamine (TEA) in the BGE was favorable, providing separation of T6P from sucrose-6-phosphate and minimizing ionization suppression. Replacing ammonium acetate with TEA enhanced T6P signal intensities more than four times. The optimized method allowed quantification of T6P in plant extracts with good linearity $\left(r^{2}>0.99\right)$ within a biologically relevant concentration range. The limit of quantification was $80 \mathrm{nM}$ in Arabidopsis extracts, corresponding to $33 \mathrm{pmol} / \mathrm{g}$ plant fresh weight. The CE-MS method was applied to the determination of T6P in seedlings from wild type (WT) Arabidopsis and mutants lacking the trehalase AtTRE1,
\end{abstract}

T. L. Delatte · G. J. de Jong • G. W. Somsen

Department of Biomedical Analysis, Utrecht University,

P.O. Box 80082, 3508 TB Utrecht, The Netherlands

T. L. Delatte $(\bowtie) \cdot H$. Schluepmann • S. C. M. Smeekens Department of Molecular Plant Physiology, Utrecht University, Padualaan 8,

$3584 \mathrm{CH}$ Utrecht, The Netherlands

e-mail: T.L.Delatte@uu.nl tre1-1, challenged with trehalose or sorbitol. T6P accumulation in tre1-1 plants grown on sorbitol was about twice the level of T6P found in WT. CE-MS is shown to be a fast and reliable technique to analyze phosphodisaccharides for seedling extracts. The low sample volume requirement of $\mathrm{CE}$ and its direct $\mathrm{MS}$ coupling makes it an attractive alternative for anion-exchange liquid chromatographyMS.

Keywords Trehalose-6-phosphate $\cdot$ Capillary electrophoresis - Mass spectrometry $\cdot$ Arabidopsis thaliana . Trehalase $\cdot$ Phosphodisaccharides

\section{Introduction}

Trehalose is a non-reducing sugar consisting of two alpha-1,1-linked glucose units. In most plants, trehalose and its precursor trehalose-6-phosphate (T6P) are present at trace levels, but it is interesting to note that in Arabidopsis, 22 genes are attributed to the trehalose synthesis pathway, including one gene encoding for a trehalase which converts trehalose into two glucose units [1]. Recently, T6P has been shown to inhibit the activity of sucrose nonfermenting-1-related protein kinase 1 (SnRK1) [2]. Other phosphorylated sugars did not show the same effect on SnRK1, highlighting T6P uniqueness. SnRK1 is a key energy and carbon metabolism regulator in plants [3] and is homologous to the mammalian AMP-activated protein kinase. SnRK1 inhibition and the link between trehalose and plant hormones position T6P centrally in the sensing of carbon availability in relation to the regulation of growth [4]. Therefore, quantifying T6P in plant extracts represents an important step towards unraveling the carbon regulatory pathway. The difficulty presented by the analysis of 
phosphodisaccharides in plant extracts is their low concentration in a complex matrix and the occurrence of isomeric compounds. For instance, the only structural difference between trehalose and sucrose - two non-reducing disaccharides present in plants - is the spatial distribution of the hydroxyl groups on the carbohydrate moieties.

The high polarity of phosphosaccharides leads to low retention in reversed phase (RP) liquid chromatography (LC). Therefore, anion-exchange chromatography (AEC) has been widely used for separation of (phospho-) carbohydrates using pulse amperometry (PAD) or mass spectrometry (MS) for detection [5-10]. AEC employs the negative charges and the hydrophilicity of the phosphocarbohydrates at high $\mathrm{pH}$ to separate the numerous species present in plant samples or other matrices [7, 11]. PAD in principle represents a very sensitive way to detect carbohydrates with limits of detection down to $200 \mathrm{nM}$. However, the selectivity of PAD is limited, hindering reliable quantitation in complex matrices, such as plant extracts. The selectivity of MS offers a large advantage providing the possibility of highly specific detection. Presently, the use of MS as a detection technique for AEC still is marginal in comparison to PAD due to the involatile salts-containing mobile phases commonly used in AEC. To allow efficient AEC-MS, the large quantities of involatile ions present in the eluent need to be removed on-line prior to MS detection using a special desalting device [7,9]. This membrane desalter exchanges sodium ions with hydrogen ions generated by the electrolysis of water and prevents the loss of analytes. This way, limits of detection of $40 \mathrm{nM}$ could be achieved for T6P analysis in plant extracts. However, regular regeneration and cleaning of the desalter are necessary for stable performance over a prolonged period of time. Furthermore, the lifetime of this module is limited.

Capillary electrophoresis (CE) is particularly suited for the separation of highly polar and charged compounds such as low molecular weight metabolites. CE separation is based on charge-to-size differences and commonly provides very narrow peaks and thus efficient separations. $\mathrm{CE}$ is a miniaturized technique requiring only minute amounts of separation buffers (i.e., background electrolytes (BGEs)) and samples. The flow rate in $\mathrm{CE}$ allows direct coupling with MS, and when volatile BGEs are used, ion suppression can be effectively avoided. Moreover, for molecules which do not absorb UV light, such as non-reducing carbohydrate and their phosphorylated analogues, MS represents a convenient, selective, and sensitive detection method. CE has shown good potential for metabolic profiling in various matrices including plant extracts [12-19]. Phosphorylated carbohydrates have been analyzed using CE-MS [12-14, 20], but so far, only Harada et al. [12] have reported T6P quantitation in a anionic metabolomic study of cell culture of Catharanthus roseus by CE-MS/ MS using a triple-quadrupole mass spectrometer and multiple reaction monitoring (MRM) for sensitive detection. They used a BGE of $50 \mathrm{mM}$ ammonium acetate ( $\mathrm{pH} 9)$, in combination with a pre-coated capillary, and needed to apply pressure on the capillary to allow CE-MS. A limit of detection of $0.16 \mu \mathrm{M}$ was reported for T6P; however, no attention was given to the separation of T6P from sucrose-6-phosphate (S6P), a known isomer commonly present in plants [11]. We set out to develop a CE-MS method capable of separating phosphorylated disaccharide isomers in plant matrix and studied its applicability for the analysis of T6P in extracts from Arabidopsis seedlings. CE-MS coupling was accomplished using a coaxial sheath liquid (SL) interface and electrospray ionization (ESI) in negative ion mode. Various BGE compositions were evaluated based on T6P MS response as well as separation potential for three phosphodisaccharide isomers viz.T6P, S6P, and lactose-1-phosphate (L1P). Furthermore, the SL composition was optimized to provide maximum T6P signals. CE-MS detection of T6P in plant matrix was investigated, and the suitability of the method was demonstrated by the analysis of T6P accumulation in Arabidopsis plants lacking the only annotated trehalase gene AtTRE1.

\section{Experimental}

\section{Reagents}

The half-strength Murashige and Skoog growth medium for plants was obtained from Duchefa (Haarlem, The Netherlands). Acetonitrile (LC-MS Chromasolv), acetic acid (puriss. LC-MS), ammonium acetate ( $\geq 99.99 \%)$, ammonium hydroxide solution (25\% LC-MS grade), chloroform (Chromasolv), diethylamine ( $\geq 99.5 \%$ ), formic acid (LC-MS grade), isopropanol (LC-MS Chromasolv), lactose-1phosphate barium salt, methanol (LC-MS Chromasolv), morpholine (redistilled, 99.5\%), piperidine (purity $>98 \%$ ), sodium hydroxide $(50 \%$ in water), sucrose-6-phosphate di-sodium ( $>98 \%$ ), T6P di-sodium ( $>95 \%$ ), and triethylamine (TEA; $\geq 99 \%$ ) were purchased from Sigma-Aldrich (Zwijndrecht, Netherlands). Acetone (99.5\% HPLC grade) was acquired from Alpha Aesar (Ward Hill, MA, USA). Highly purified water obtained with a Milli-Q system (Millipore, Bedford, MA, USA) was used during this work.

Plant material

Seed from the line Salk_147073.21.10.x was ordered at NASC (European Arabidopsis Stock Centre), and then 
grown on soil for reproduction and genetic analyses. Polymerase chain reaction (PCR) analyses on DNA from the individual plants to confirm homozygous knockout in AtTRE1 were performed by using two nested primer pairs spanning the insertion: ForwardAtTRE1LP1 5'-TGAATTG GATCTCCTTATGGC-3', ReverseAtTRE1RP1 5'-AGT GACGAGTTTGGTTGTTGC-3' and ForwardAtTRE1LP2 5'-TGTGATTCCATCTCTTCATCC-3', ReverseAtTRE1RP2 5 '-GTGTCTGTGTCCGAGTCCAAC-3'. The insertion was confirmed by sequencing the flanking region amplified by PCR using one primer from the T-DNA LbB1 5'GCGTGGACCGCTTGCTGAACT-3' and the primer AtTRE1RP1 5'-AGTGACGAGTTTGGTTGTTGC-3'. Quantitative reverse transcription-PCR of Attrel mRNA revealed that Attrel gene expression was below reliable detection in seedlings (Q-PCR primers: 5'-AGCGAGA GAGAAAGCGTTTC-3' and 5'-CCTTCCATGTCTCA GATTC-3', expression was calculated relative to AtPP2A (Atlg10430) regulatory subunit a housekeeping gene in Arabidopsis [21]). Together with the increased seedling sensitivity to trehalose in the medium, it was concluded that the line was a knockout for AtTRE1 and will be henceforth called tre1-1.

Seed from Arabidopsis wild type (WT) accession Col.0 and tre1-1 mutants were sterilized using chlorine [22]. Seedlings were grown in $10 \mathrm{~mL}$ of liquid half strength MS medium, pH 5.7 (Duchefa) at $22{ }^{\circ} \mathrm{C}$ in a growth cabinet (Snijders Scientific, Tilburg, The Netherlands) under constant fluorescent light $\left(100 \mu \mathrm{mol} \mathrm{m} \mathrm{m}^{-2} \mathrm{~s}^{-1}\right)$ with continuous rotary shaking $(180 \mathrm{rpm})$. After 7 days, seedlings were treated for $4 \mathrm{~h}$ with $100 \mathrm{mM}$ - final concentration - of either sorbitol or trehalose. Sorbitol was used here as an osmotic control. Seedlings were harvested on a sieve, rapidly rinsed, dry-blotted, and weighed to $50 \mathrm{mg}$ fresh weight, then flash-frozen in liquid nitrogen and stored at $-80{ }^{\circ} \mathrm{C}$ before further use.

\section{Sample preparation}

Plant samples were prepared as described by Delatte et al. [7]. Briefly, the protocol was as follows. The samples were frozen in liquid nitrogen and stored at $-80{ }^{\circ} \mathrm{C}$. Frozen samples were ground in liquid nitrogen into a fine powder and extracted using a liquid/liquid extraction (LLE) procedure. The LLE was performed by dispersing the seedling powder with $500 \mu \mathrm{L}$ of chloroform/acetonitrile $(3: 7, v / v)$. The mixture was kept at $-20{ }^{\circ} \mathrm{C}$ and regularly shaken for $2 \mathrm{~h}$. Phosphorylated sugars were extracted twice with $400 \mu \mathrm{L}$ water at $4{ }^{\circ} \mathrm{C}$ from the organic phase by vigorous shaking for $30 \mathrm{~s}$ followed by $5 \mathrm{~min}$ of centrifugation at maximum speed. The aqueous supernatants were pooled and then evaporated to dryness using a centrifugal vacuum dryer at room temperature. The dried samples were reconstituted in water prior to solid-phase extraction (SPE), which was performed with Oasis MAX cartridges (Waters, Milford, MA, USA). Prior to loading, the samples were diluted ten times with water. The SPE column was washed with $1 \mathrm{~mL}$ of methanol and subsequently with $1 \mathrm{~mL}$ of water. T6P was desorbed with $1 \mathrm{~mL}$ of $2 \%$ formic acid $(v / v)$ in methanol, and the eluate was dried under nitrogen. The dried samples were stored at $-80{ }^{\circ} \mathrm{C}$ until analysis. Prior to analysis, the extracts were reconstituted in $200 \mu \mathrm{L}$ water. T6P recoveries with this protocol were above $80 \%$ [7].

\section{Instrumentation}

CE separations were carried out on a P/ACE MDQ (Beckman Coulter, Fullerton, CA, USA) instrument monitored with 32 Karat Software version 7.0 (Beckman Coulter). Fused silica capillaries were from Polymirco (Eerbeek, The Netherlands), had a 50- $\mathrm{mm}$ i.d. (365 $\mu \mathrm{m}$, o.d.), and a length of $132 \mathrm{~cm}$. The separation was carried out at normal polarity $(30 \mathrm{kV})$ at $15{ }^{\circ} \mathrm{C}$ using an uncoated capillary. Sample injection was performed by hydrodynamic injection for $40 \mathrm{~s}$ with a pressure of 3 psi (ca. $85 \mathrm{~nL}$ ). A new capillary was rinsed with methanol for $5 \mathrm{~min}$, Milli-Q water $5 \mathrm{~min}$ (20 psi), $0.5 \mathrm{M} \mathrm{NaOH}$ for $15 \mathrm{~min}$ (20 psi), and Milli-Q water for $15 \mathrm{~min}$ (40 psi). In between runs, the capillary was flushed with Milli-Q water for $3 \mathrm{~min}$ (50 psi), acetic acid $(10 \% ; v / v$ at $20 \mathrm{psi})$, and BGE for $5 \mathrm{~min}(15 \mathrm{psi})$. At the end of the day, the capillary was washed with BGE for $5 \mathrm{~min}$, acetic acid $(10 \% ; v / v), 0.5 \mathrm{M} \mathrm{NaOH}$ for $2 \mathrm{~min}$, and finally, Milli-Q water for $10 \mathrm{~min}$, all steps performed using a pressure of 40 psi.

The detector was a MicroTOF-QII mass spectrometer equipped with ESI source (Bruker-Daltonics, Bremen, Germany) and a SL electrospray interface (Agilent Technologies, Amstelveen, The Netherlands). Nitrogen was used as drying gas at $170{ }^{\circ} \mathrm{C}$ and a flow-rate of $4 \mathrm{~L} / \mathrm{min}$. Nitrogen sheath gas for ESI was supplied at 4 psi. The SL was delivered to the ESI interface by a LC 10ADvp (Shimadzu, Kyoto, Japan) pump equipped with a 1:100 flow splitter (Agilent Technologies) at $3 \mu \mathrm{L} / \mathrm{min}$. ESI was operated in negative mode with an ESI voltage of $3.7 \mathrm{kV}$. The $m / z$ range scanned was from 50 to 500. Electropherograms were constructed using DataAnalysis version 4.0 (BrukerDaltonics) with extracted ion chromatogram function selecting $m / z 421.15 \pm 0.02$. Peak area and signal-to-noise ratio were obtained with DataAnalysis version 4.0 (Bruker-Daltonics).

Method validation and T6P quantitation

The linearity of the method was checked by measuring six concentrations of T6P between $80 \mathrm{nM}$ and $80 \mu \mathrm{M}$. These measurements were performed using water and spiked 
matrix. The T6P peak was identified as described in Delatte et al. [7]. Endogenous T6P level was quantified by averaging data from six analyses of sorbitol-grown Arabidopsis extracts; subsequently, this level was subtracted from measurements of spiked matrix. The lower limit of quantification (LOQ) was determined by repeatedly $(n=9)$ analyzing decreasing concentrations of T6P by CE-MS. The lowest concentration that still had an RSD below $10 \%$ was denoted as LOQ. Accuracy of the CE-MS method was determined with matrix samples, spiked before injection, with three T6P concentrations $(100 \mathrm{nM}$, $4 \mu \mathrm{M}$, and $6 \mu \mathrm{M})$. The reproducibility of the method was assessed by injecting a 100-nM T6P spike sample over the course of 3 days of measurements. The quantitation of T6P in plant extracts was inferred from the calibration curve. Five biological replicates were analyzed for each genotype and growth condition.

\section{Results and discussion}

\section{Optimization of BGE}

Plants hold an array of phosphosaccharides in their metabolism. Separation is crucial to be able to reliably differentiate and quantitate specific sugar phosphates present in plant samples. Increase the $\mathrm{CE}$ selectivity among phosphorylated carbohydrates, and their isomers in particular, it is important to induce extra charge on these molecules. Therefore, BGEs of high $\mathrm{pH}$ were selected for $\mathrm{CE}$ analysis. The low abundance of these metabolites in plant extracts also requires sensitive detection.

We first assessed the impact of BGEs on the MS response of T6P by infusing solutions of T6P $(3 \mathrm{mM})$ in various BGEs. Infusion was performed at $50 \mathrm{psi}$, and the SL was water-isopropanol-ammonium hydroxide (50:50:1, $v / v / v)$. The T6P signal was compared with the response obtained for T6P in water. The BGEs studied were $50 \mathrm{mM}$ TEA (pH 11.3), 50 mM TEA (pH 11.0, adjusted with acetic acid), $50 \mathrm{mM}$ TEA (pH 11.0, adjusted with formic acid), $30 \mathrm{mM}$ morpholine ( $\mathrm{pH} 8.6$ ), and $50 \mathrm{mM}$ ammonium acetate ( $\mathrm{pH}$ 9.0, adjusted with ammonium hydroxide). The strongest signal reduction observed for T6P relative to water was with a BGE of $50 \mathrm{mM}$ ammonium acetate (pH 9.0; Fig. 1). Remarkably, ammonium acetate is widely used in CE-MS for metabolomic purposes, including analysis of phosphorylated carbohydrates [15-17, 23-31]. In our study, this BGE generated up to $80 \%$ of signal reduction. The highest signal for T6P was obtained with TEA as a BGE. Diethylamine (50 mM, pH 11.0) was also tested as a BGE showing performances similar to TEA, except for a worse reproducibility in response and

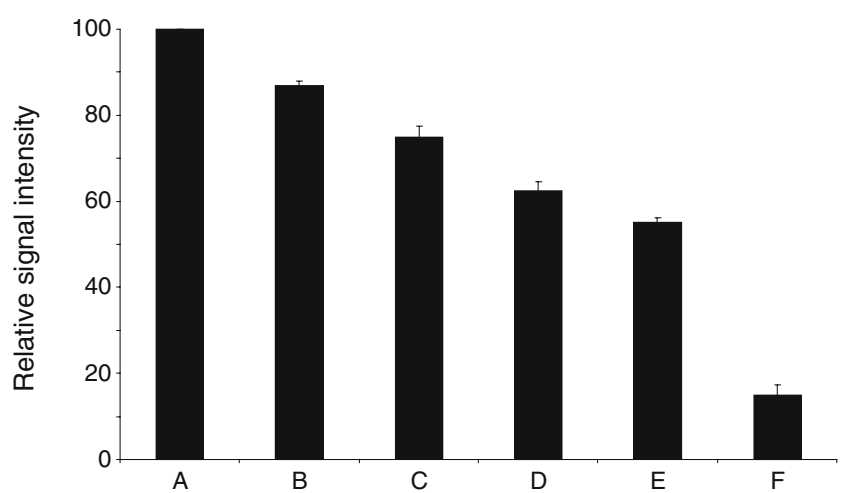

Fig. 1 Relative signal intensity obtained for T6P (3 mM) during infusion in various solvents: $A$ water, $B 50 \mathrm{mM}$ TEA (pH 11.3), $C$ $50 \mathrm{mM}$ TEA (pH 11.0 adjusted with acetic acid), $D 50 \mathrm{mM}$ TEA (pH 11.0 adjusted formic acid), $E 30 \mathrm{mM}$ morpholine (pH 8.3), and $F$ $50 \mathrm{mM}$ ammonium acetate $(\mathrm{pH} 9.0$ adjusted with ammonium hydroxide). Values are means $\pm \mathrm{SE}(n=6)$ randomized independent measurements

migration time. When formic or acetic acid was used to lower the $\mathrm{pH}$ of the TEA BGE, the signal intensity was reduced. A BGE of $30 \mathrm{mM}$ morpholine also suppressed the T6P signal, but not as strongly as ammonium acetate did. Based on these results, we conclude that acetate and formate may adversely affect T6P ionization. Furthermore, the choice of the positive co-ion of the BGE seems to be important in order to achieve optimum T6P signals in negative mode ESI.

To evaluate the separation power of the CE system, we used a test mixture of the isomeric phosphodisaccharides L1P, S6P, and T6P during our method development. BGEs of $30 \mathrm{mM}$ morpholine ( $\mathrm{pH} 8.5$ ), $50 \mathrm{mM}$ ammonium acetate (pH 9.0), $50 \mathrm{mM}$ piperidine ( $\mathrm{pH} 11.5$ ), and $50 \mathrm{mM}$ TEA $(\mathrm{pH}$ 11.3) were tested. CE-MS using morpholine as BGE provided the shortest migration time, but no separation was obtained for the three test isomers (Fig. 2a). Using $50 \mathrm{mM}$ ammonium acetate as BGE did not improve the separation (Fig. 2b). Using $50 \mathrm{mM}$ piperidine as BGE, S6P was separated from T6P and L1P, which co-migrated (Fig. 2c). With $50 \mathrm{mM}$ TEA, a baseline separation of the three isomers was obtained (Fig. 2d), also providing the highest MS response from the isomers when compared to the other BGEs. Based on the separation of the test isomers and the obtained signal intensity, TEA was selected as optimal BGE for CE-MS.

We further studied the influence of the TEA concentration in the BGE on resolution (Fig. 3). Concentrations from 5 to $50 \mathrm{mM}$ of TEA were used. From the results, it was clear that a minimum concentration of $50 \mathrm{mM}$ was required to induce the separation of the three isomers. Increasing the concentration of TEA in the BGE induced slower migration, providing a better resolution, but it also decreased the peak height of the isomers present in the test mixture. 


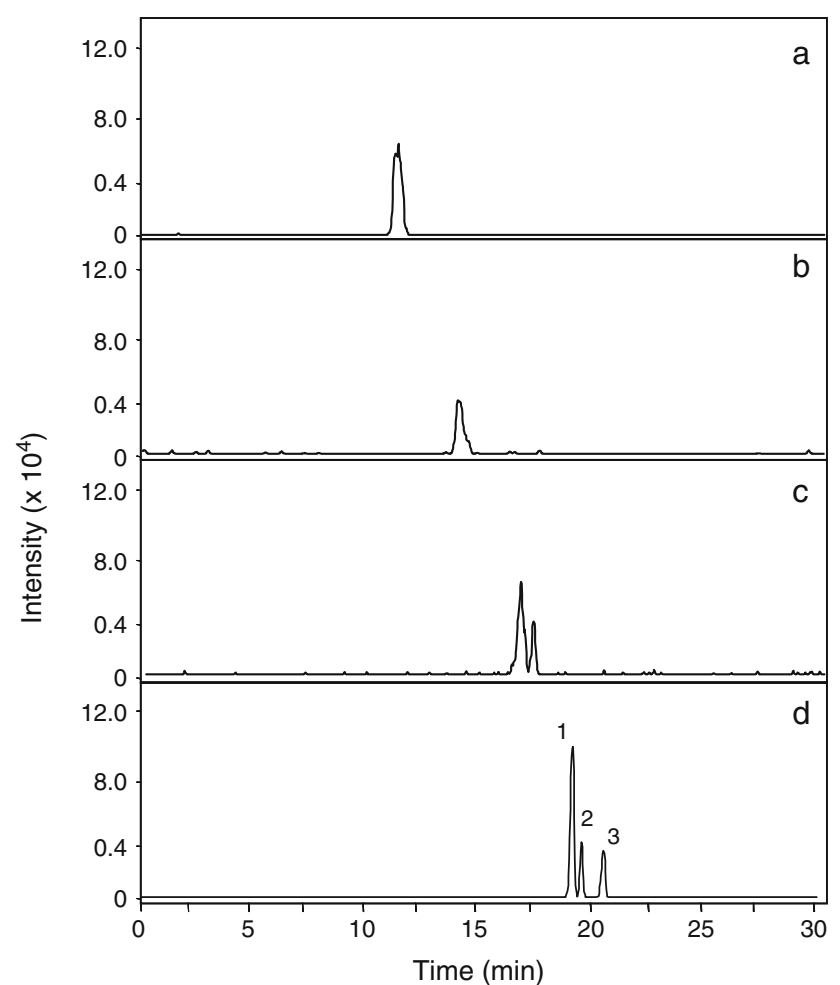

Fig. 2 Extracted ion electropherograms $(\mathrm{m} / \mathrm{z} 421.15 \pm 0.02)$ obtained during CE-MS of a mixture of L1P, T6P, and S6P $(800 \mathrm{nM}$ in water each). BGE: $a 30 \mathrm{mM}$ morpholine (pH 8.5), b $50 \mathrm{mM}$ ammonium acetate ( $\mathrm{pH} 9.0), c 50 \mathrm{mM}$ piperidine ( $\mathrm{pH} 11.5)$, and $d 50 \mathrm{mM}$ TEA (pH 11.3). Peaks corresponding to L1P, T6P, and S6P are labeled 1, 2, and 3 , respectively

In an attempt to further improve the separation of the phosphodisaccharides present in our test mixture, we also studied the effect of the addition of organic solvents to the BGE. Four solvents were selected: acetone, acetonitrile, isopropanol, and methanol. Each of these compounds was introduced to the $50-\mathrm{mM}$ TEA BGE in amounts ranging from $5 \%$ to $40 \%(v / v)$, and CE-MS of the test mixture was

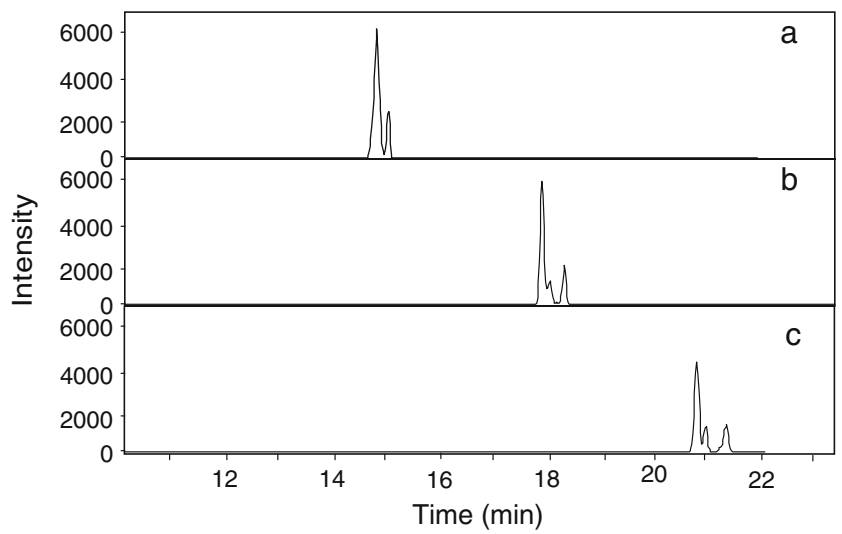

Fig. 3 Extracted ion electropherograms $(\mathrm{m} / \mathrm{z} 421.15 \pm 0.02)$ obtained during CE-MS of a mixture of L1P, T6P, and S6P $(600 \mathrm{nM}$ in water each). TEA concentration in BGE: $a 5, b 25$, and $c 50 \mathrm{mM}$ performed. Acetone, isopropanol, and methanol did not improve the separation, but significantly increased the migration times and decreased the peak heights of the analytes. Addition of $5 \%(v / v)$ acetonitrile to the BGE caused an average increase in peak height by a factor of 1.2 while maintaining separation. Addition of $10 \%(v / v)$ acetonitrile to the BGE did not provide higher signals nor better separation when compared with $5 \%$. The presence of organic solvent in the BGE reduced its conductivity. A separation voltage of $30 \mathrm{kV}$ caused a current of ca. $3 \mu \mathrm{A}$ only, thus minimizing joule-heating effects. The average electroosmotic mobility under these conditions was $5.38 \times$ $10^{-8} \mathrm{~m}^{2} / \mathrm{Vs}$. Based on these results, a BGE of $50 \mathrm{mM}$ TEA $(\mathrm{pH} 11.3)$ with $5 \%(v / v)$ acetonitrile was selected for the analysis of T6P.

Sheath liquid

The SL used for CE-MS replaces the terminating BGE vial of a classical CE system. It allows the electrical connection necessary for $\mathrm{CE}$ separation and for maintaining a constant electrospray. Furthermore, the constitution of the SL may enhance the formation of analyte ions during ESI. Normally, the SL is made up of organic solvents and water in various ratios plus an additive providing conductivity and the adequate $\mathrm{pH}$ for ionization. Firstly, we compared formic acid, acetic acid, ammonium acetate, DEA, and TEA as ionization additives for the SL. For each additive tested, $0.1 \%(v / v)$ was added to water-isopropanol 50:50 $(v / v)$. The SL was tested by monitoring the signal of a solution of $0.8 \mu \mathrm{M} \mathrm{T} 6 \mathrm{P}$, which was infused at $50 \mathrm{psi}$. The SL flow rate was set at $3 \mu \mathrm{L} / \mathrm{min}$. Formic and acetic acid added in the SL did not improve the signal obtained for T6P when compared with ammonium hydroxide as SL additive. Ammonium acetate produced a signal intensity for T6P that was about 30\% lower than the one obtained with ammonium hydroxide. However, addition of DEA and TEA to the SL enhanced the T6P signal by a factor 1.8 with respect to ammonium hydroxide. These findings were consistent with the results observed during BGE optimization. Increase of the TEA and DEA concentrations in the SL up to $0.5 \%$ did not further enhance the T6P signal, whereas with concentrations below $0.1 \%$, lower signals were obtained. A concentration of $0.1 \%$ TEA was selected for the SL.

As organic solvent component of the SL, methanol, isopropanol, and acetonitrile were tested at concentrations of $25 \%, 50 \%$, and $75 \%(v / v)$. A concentration of $50 \%(v / v)$ isopropanol appeared to provide optimal ionization conditions for T6P. At equal concentration, methanol was less effective than isopropanol to enhance T6P signal. SLs with acetonitrile provided a T6P signal that was about $50 \%$ lower then the average signal obtained with SLs containing alcohols, although acetonitrile was 
favorable as a BGE additive. A SL of isopropanol-waterTEA (50:50:0.1, $v / v / v)$ was used for further experiments.

\section{System performance}

CE-MS of T6P was conducted with a BGE of $50 \mathrm{mM}$ TEA $(\mathrm{pH} 11.3)$ containing $5 \%(v / v)$ acetonitrile and a SL of water-isopropanol-TEA $(50: 50: 0.1, v / v / v)$. To verify that we could detect T6P and separate it from S6P at endogenous levels, we analyzed WT Arabidopsis seedling from 7-day-old seedlings grown in liquid medium, after a 4-h treatment with sorbitol (Fig. 4). We obtained good separation for peaks corresponding to T6P and S6P. The absence of T6P in Arabidopsis is embryo lethal [32]; thus, it is impossible to obtain seedling extract without endogenous levels of T6P. Therefore, for quantitative purposes, endogenous levels of T6P were obtained by averaging the analysis of three blank sorbitol-grown seedling extracts, providing an endogenous T6P level which was subtracted from levels found in spiked matrix samples. The performance of the system was tested by analyzing T6P at various concentrations $(80 \mathrm{nM}-8 \mu \mathrm{M})$ in water and in Arabidopsis seedling extracts by CE-MS. From these measurements, extracted ion electropherograms were generated, and calibration curves were constructed by plotting the T6P peak area versus T6P concentrations (Table 1). An excellent linearity of the CE-MS method for biologically relevant concentrations in plants was observed. The plots obtained for the water standards and spiked matrix were very similar, with less than $3 \%$ deviation between the slopes. This means that interferences of the T6P signal by matrix compounds were minimal, indicating proper separation by CE. The accuracy of the CE-MS method was investigated by analyzing $(n=3)$ T6P spiked seedling extracts (100 nM, $4 \mu \mathrm{M}$, and $6 \mu \mathrm{M}$ ); the T6P concentration present in these spiked samples was inferred with the calibration curve. These results were then compared to the known amounts added to the extracts yielding accuracies of $92 \%$, $96 \%$, and $102 \%$, respectively, for the CE-MS method. The

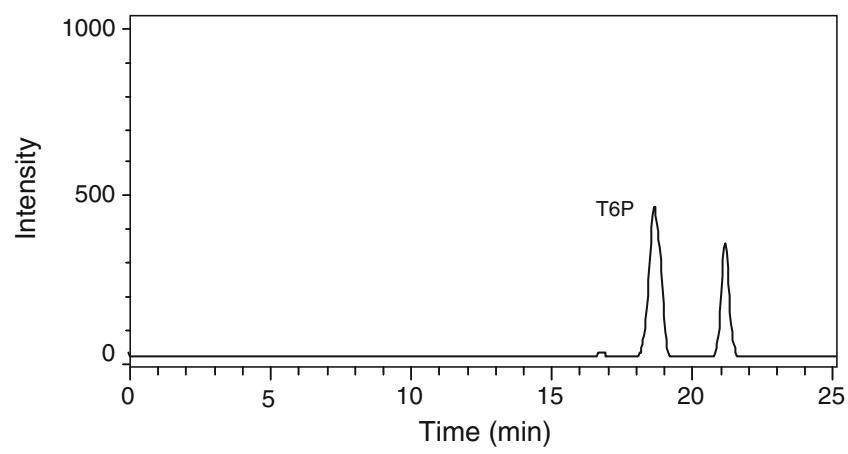

Fig. 4 Extracted ion electropherograms $(\mathrm{m} / \mathrm{z}$ 421.15 \pm 0.02$)$ obtained during CE-MS of Arabidopsis seedlings extracts resulting from plants induced for $4 \mathrm{~h}$ with $100 \mathrm{mM}$ of sorbitol
Table 1 Linearity for T6P as determined by CE-MS in water and plant matrix

\begin{tabular}{lccc}
\hline Conditions & Regression equation $^{\mathrm{a}, \mathrm{b}}$ & Range & $R^{2}$ \\
\hline Aqueous & $y=16.17 x-170.78$ & $\begin{array}{c}80 \mathrm{nM}-8 \mu \mathrm{M} \\
(6 \text { points })\end{array}$ & 0.9998 \\
standards & $\begin{array}{c}80 \mathrm{nM}-8 \mu \mathrm{M} \\
\text { (6 points) }\end{array}$ & 0.9979 \\
Plant extracts & $y=15.98 x-259.20$ & \\
\hline
\end{tabular}

a $y$ represents the area of the T6P peak

${ }^{\mathrm{b}} x$ represents the injected T6P concentration in nanomolar

limit of quantitation for $\mathrm{T} 6 \mathrm{P}$ in water standards was determined to be $80 \mathrm{nM}(n=9$; peak area RSD, $<10 \%$ ). The lowest T6P concentration measured in WT seedlings grown on sorbitol was measured at $93 \mathrm{nM}$, T6P. The obtained concentration of T6P was then converted in nanomoles per gram fresh weight ( $\mathrm{g} \mathrm{FW}$ ) using the weighed sample mass and taking into account the various extraction steps, resulting in a limit of quantification of $33 \mathrm{pmol} / \mathrm{g} \mathrm{FW}$ for Arabidopsis seedling extracts. This limit of quantitation in plant extracts is similar to results obtained with AEC-MS and lower than for AEC-PAD [7]. It should be noted that the sample volumes injected in AEC-MS and AEC-PAD are more than 500 times larger, indicating an excellent sensitivity in terms of absolute detected T6P amounts for the present CE-MS method. The obtained limit of detection for T6P in plant extracts is also lower than reported for CE-MS/MS applying MRM with a triple quadrupole mass spectrometer [12]. As the latter detection principle is more sensitive than TOF-MS, the lower limit of detection for T6P should be attributed to the use of TEA in the BGE instead of ammonium acetate.

The reproducibility of the CE-MS method was investigated by analysis of a spiked seedling extract (100 nM), injected six times over three consecutive analysis days. Using these results, the RSD for the T6P peak area was below $4 \%$ and migration time below $6 \%$.

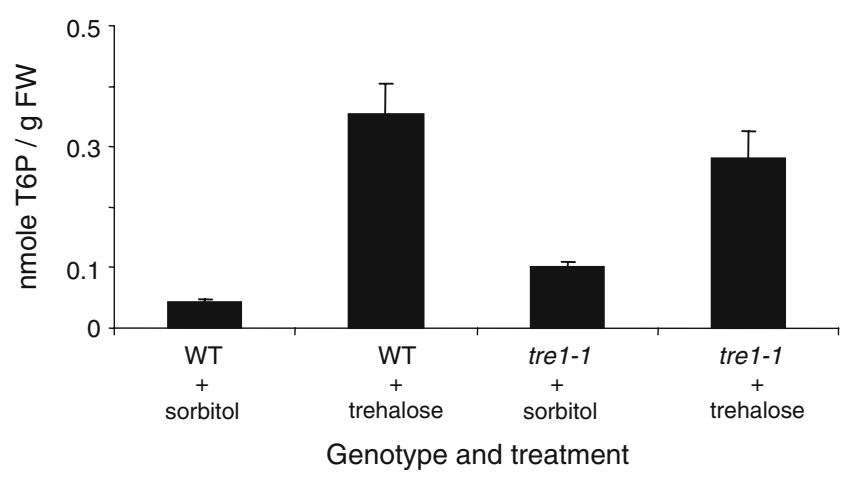

Fig. 5 T6P quantification in WT and tre1-1 Arabidopsis thaliana seedlings induced for $4 \mathrm{~h}$ with $100 \mathrm{mM}$ sorbitol or trehalose. Seedlings were grown for 7 days in liquid medium prior to induction. Each bar represents the average of five biological replicates $( \pm \mathrm{SE})$ 
T6P in planta quantitation

To demonstrate applicability of the CE-MS method, we set out to analyze T6P accumulation in seedlings lacking AtTRE1, the only known trehalase gene in Arabidopsis. To test if AtTRE1 affects the accumulation of T6P in seedlings, WT and tre 1-1 seedlings were analyzed after $4 \mathrm{~h}$ treatment with trehalose or sorbitol. Sorbitol was used here as an osmoticum control, to account for the change in osmotic strength in the growth medium. The T6P content from WT and tre1-1 was analyzed using the CE-MS method described above. The injections were randomized and performed over a period of 3 days (Fig. 5). The WT seedlings treated with sorbitol had a total T6P quantity of less than $0.04 \mathrm{nmol} / \mathrm{g}$ of plant fresh weight, whereas for the same treatment, tre 1-1 plants had twice as much T6P present. Interestingly, when the two genotypes were challenged with a 4-h treatment of $100 \mathrm{mM}$ trehalose, the total T6P accumulation was not significantly different between the WT and tre $1-1$. We conclude that AtTRE1 has no effect on T6P accumulation when seedlings are treated with $100 \mathrm{mM}$ trehalose. AtTRE1, however, may have an effect on T6P accumulation in seedlings after osmotic changes in the environment as we measure a doubling of T6P concentration in tre 1-1 seedlings compared to WT after treatment with $100 \mathrm{mM}$ sorbitol. Further study of T6P dynamics in tre 1-1 over time and in different conditions would provide a better understanding of the role of this enzyme for T6P steady state.

\section{Conclusions}

In this paper, we demonstrate that CE-MS can be successfully used for the separation of phosphodisaccharides in plant extracts. Ammonium acetate, which is a popular BGE for metabolomic analysis by CE-MS, was shown to perform poorly for separating T6P from its isomers and for T6P response. In contrast, TEA showed better results for separation and T6P signal intensity. The matrix effect observed for seedling extract was minimal, leading to an accuracy above $90 \%$ in the concentration range relevant for in planta T6P quantitation. The linearity $\left(r^{2}>0.999\right)$ and limit of quantification $(80 \mathrm{nM})$ were also adequate for the application at hand. The method was applied to measure the accumulation of T6P in Arabidopsis seedlings lacking the only trehalase enzyme annotated in the Arabidopsis genome. The trel-1 seedlings showed a twofold higher T6P level than their corresponding WT after $4 \mathrm{~h}$ treatment with $100 \mathrm{mM}$ sorbitol (osmoticum control). CE-MS was shown to be fast and reliable for the separation of phosphodisaccharides present in plants and quantification of T6P. The low sample volume require- ment of $\mathrm{CE}$ and its direct $\mathrm{MS}$ coupling make it an attractive alternative for AEC-MS for T6P analysis in plant extracts.

Acknowledgment This work was financially supported by the Netherlands Organization for Scientific Research (ECHO project 700.56.011)

Open Access This article is distributed under the terms of the Creative Commons Attribution Noncommercial License which permits any noncommercial use, distribution, and reproduction in any medium, provided the original author(s) and source are credited.

\section{References}

1. Lunn JE (2007) Gene families and evolution of trehalose metabolism in plants. Funct Plant Biol 34(6):550-563

2. Zhang YH, Primavesi LF, Jhurreea D, Andralojc PJ, Mitchell RAC, Powers SJ, Schluepmann H, Delatte T, Wingler A, Paul MJ (2009) Inhibition of snf1-related protein kinase1 activity and regulation of metabolic pathways by trehalose-6-phosphate. Plant Physiol 149(4):1860-1871

3. Hanson J, Smeekens S (2009) Sugar perception and signaling - an update. Curr Opin Plant Biol 12(5):562-567

4. Smeekens S, Ma JK, Hanson J, Rolland F (2010) Sugar signals and molecular networks controlling plant growth. Curr Opin Plant Biol 13(3):274-279

5. Lunn JE, Feil R, Hendriks JHM, Gibon Y, Morcuende R, Osuna D, Scheible WR, Carillo P, Hajirezaei MR, Stitt M (2006) Sugarinduced increases in trehalose 6-phosphate are correlated with redox activation of ADPglucose pyrophosphorylase and higher rates of starch synthesis in Arabidopsis thaliana. Biochem $\mathrm{J}$ 397:139-148

6. Delatte T, Trevisan M, Parker ML, Zeeman SC (2005) Arabidopsis mutants atisa1 and atisa2 have identical phenotypes and lack the same multimeric isoamylase, which influences the branch point distribution of amylopectin during starch synthesis. Plant J 41 (6):815-830

7. Delatte TL, Selman MHJ, Schluepmann H, Somsen GW, Smeekens SCM, de Jong GJ (2009) Determination of trehalose6-phosphate in Arabidopsis seedlings by successive extractions followed by anion exchange chromatography-mass spectrometry. Anal Biochem 389(1):12-17

8. Delatte T, Umhang M, Trevisan M, Eicke S, Thorneycroft D, Smith SM, Zeeman SC (2006) Evidence for distinct mechanisms of starch granule breakdown in plants. J Biol Chem 281 (17):12050-12059

9. Chataigne G, Couderc F, Poinsot V (2008) Polysaccharides analysis of sinorhizobial capside by on-line anion exchange chromatography with pulsed amperometric detection and mass spectrometry coupling. J Chromatogr A 1185(2):241-250

10. Bruggink C, Wuhrer M, Koeleman CAM, Barreto V, Liu Y, Pohl C, Ingendoh A, Hokke CH, Deelder AM (2005) Oligosaccharide analysis by capillary-scale high-pH anion-exchange chromatography with on-line ion-trap mass spectrometry. J Chromatogr B Anal Technol Biomed Life Sci 829(1-2):136-143

11. Chen SA, Hajirezaei M, Peisker M, Tschiersch H, Sonnewald U, Bornke F (2005) Decreased sucrose-6-phosphate phosphatase level in transgenic tobacco inhibits photosynthesis, alters carbohydrate partitioning, and reduces growth. Planta 221 (4):479-492

12. Harada K, Ohyama Y, Tabushi T, Kobayashi A, Fukusaki E (2008) Quantitative analysis of anionic metabolites for Catharanthus 
roseus by capillary electrophoresis using sulfonated capillary coupled with electrospray ionization-tandem mass spectrometry. J Biosci Bioeng 105(3):249-260

13. Jumtee K, Okazawa A, Harada K, Fukusaki E, Takano M, Kobayashi A (2009) Comprehensive metabolite profiling of phyA phyB phyC triple mutants to reveal their associated metabolic phenotype in rice leaves. J Biosci Bioeng 108(2):151-159

14. Harada K, Fukusaki E, Kobayashi A (2006) Pressure-assisted capillary electrophoresis mass spectrometry using combination of polarity reversion and electroosmotic flow for metabolomics anion analysis. J Biosci Bioeng 101(5):403-409

15. Hirayama A, Kami K, Sugimoto M, Sugawara M, Toki N, Onozuka H, Kinoshita T, Saito N, Ochiai A, Tomita M, Esumi H, Soga T (2009) Quantitative metabolome profiling of colon and stomach cancer microenvironment by capillary electrophoresis time-of-flight mass spectrometry. Cancer Res 69(11):4918-4925

16. Timischl B, Dettmer K, Kaspar H, Thieme M, Oefner PJ (2008) Development of a quantitative, validated capillary electrophoresistime of flight-mass spectrometry method with integrated highconfidence analyte identification for metabolomics. Electrophoresis 29(10):2203-2214

17. Siren H, Seppanen-Laakso T, Oresic M (2008) Capillary electrophoresis with UV detection and mass spectrometry in method development for profiling metabolites of steroid hormone metabolism. J Chromatogr B Anal Technol Biomed Life Sci 871(2):375-382

18. Ramautar R, Mayboroda OA, Derks RJE, van Nieuwkoop C, van Dissel JT, Sornsen GW, Deelder AM, de Jong GJ (2008) Capillary electrophoresis-time of flight-mass spectrometry using noncovalently bilayer-coated capillaries for the analysis of amino acids in human urine. Electrophoresis 29(12):2714-2722

19. Ramautar R, Torano JS, Somsen GW, de Jong GJ (2010) Evaluation of CE methods for global metabolic profiling of urine. Electrophoresis 31(14):2319-2327

20. Hui JPM, Yang J, Thorson JS, Soo EC (2007) Selective detection of sugar phosphates by capillary electrophoresis/mass spectrometry and its application to an engineered $E$. coli host. Chembiochem 8 (10): $1180-1188$

21. Czechowski T, Stitt M, Altmann T, Udvardi MK, Scheible WR (2005) Genome-wide identification and testing of superior reference genes for transcript normalization in Arabidopsis. Plant Physiol 139(1):5-17
22. Clough SJ, Bent AF (1998) Floral dip: a simplified method for Agrobacterium-mediated transformation of Arabidopsis thaliana. Plant J 16(6):735-743

23. Arvidsson B, Johannesson N, Citterio A, Righetti PG, Bergquist J (2007) High throughput analysis of tryptophan metabolites in a complex matrix using capillary electrophoresis coupled to time-offlight mass spectrometry. J Chromatogr A 1159(1-2):154-158

24. Edwards JL, Chisolm CN, Shackman JG, Kennedy RT (2006) Negative mode sheathless capillary electrophoresis electrospray ionization-mass spectrometry for metabolite analysis of prokaryotes. J Chromatogr A 1106(1-2):80-88

25. Itoh A, Ohashi Y, Soga T, Mori H, Nishioka T, Tomita M (2004) Application of capillary electrophoresis-mass spectrometry to synthetic in vitro glycolysis studies. Electrophoresis 25(13):1996-2002

26. Peterson ZD, Collins DC, Bowerbank CR, Lee ML, Graves SW (2002) Determination of catecholamines and metanephrines in urine by capillary electrophoresis-electrospray ionization-time-offight mass spectrometry. J Chromatogr B Anal Technol Biomed Life Sci 776(2):221-229

27. Soga T, Igarashi K, Ito C, Mizobuchi K, Zimmermann HP, Tomita M (2009) Metabolomic profiling of anionic metabolites by capillary electrophoresis mass spectrometry. Anal Chem 81 (15):6165-6174

28. Soga T, Ishikawa T, Igarashi S, Sugawara K, Kakazu Y, Tomita M (2007) Analysis of nucleotides by pressure-assisted capillary electrophoresis-mass spectrometry using silanol mask technique. J Chromatogr A 1159(1-2):125-133

29. Soga T, Ohashi Y, Ueno Y, Naraoka H, Tomita M, Nishioka T (2003) Quantitative metabolome analysis using capillary electrophoresis mass spectrometry. J Proteome Res 2(5):488-494

30. Soga T, Ueno Y, Naraoka H, Matsuda K, Tomita M, Nishioka T (2002) Pressure-assisted capillary electrophoresis electrospray ionization mass spectrometry for analysis of multivalent anions. Anal Chem 74(24):6224-6229

31. Ullsten S, Danielsson R, Backstrom D, Sjoberg P, Bergquist J (2006) Urine profiling using capillary electrophoresis-mass spectrometry and multivariate data analysis. J Chromatogr A 1117(1):87-93

32. Eastmond PJ, van Dijken AJH, Spielman M, Kerr A, Tissier AF, Dickinson HG, Jones JDG, Smeekens SC, Graham IA (2002) Trehalose-6-phosphate synthase 1, which catalyses the first step in trehalose synthesis, is essential for Arabidopsis embryo maturation. Plant J 29(2):225-235 